\title{
Biosensor de Glucosa basado en un Biocompósito disperso de Grafito-Epoxi-Platino-Glucosa Oxidasa
}

\author{
José L. Montañez ${ }^{(1)}$, Emma G. Ramos ${ }^{(2)}$, Salvador Alegret ${ }^{(3)}$ y Raúl J. Delgado $^{(4)}$ \\ (1) Centro Interdisciplinario de Investigación para el Desarrollo Integral Regional (CIIDIR-IPN- \\ $\mathrm{MICH}$ ). Justo Sierra 28, C.P. 59510. Jiquilpan, Mich.-México (e-mail: montasoto@yahoo.com.mx) \\ (2) Depto. de Biotecnología y Bioingeniería, Centro de Investigación y Estudios Avanzados, \\ Instituto Politécnico Nacional, (CINVESTAV-IPN), Avenida IPN 2508, C.P. 07300, México, D.F.- \\ México
}

(3) Departamento de Química, Grupo de Sensores y Biosensores, Universidad Autónoma de Barcelona, 08193 Bellaterra, Cataluña (España) (e-mail: salvador.alegret@uab.cat)

(4) Centro de Investigación en Biotecnología Aplicada, Instituto Politécnico Nacional, Km 1.5 Carretera Tecuexcomac-Tepetila, C.P. 90600, Tlaxcala, México. (e-mail:

rdmacuil@yahoo.com.mx)

Recibido May. 04, 2010; Aceptado Jun. 04, 2010; Versión Final recibida Jun. 23, 2010

\begin{abstract}
Resumen
El objetivo del trabajo consistió en desarrollar un biosensor de glucosa basado en las propiedades electroquímicas de un compósito grafito-epoxi-platino-glucosa oxidasa. La industria de alimentos y bebidas demanda métodos analíticos rápidos, precisos y confiables para evaluar y asegurar la calidad de sus productos y optimizar sus procesos. El desarrollo de biosensores enzimáticos amperométricos representa una opción viable que satisface estos requerimientos. El potencial de trabajo y la caracterización de la respuesta del biosensor desarrollado se determinaron por voltamperometría de barrido lineal y amperometría, respectivamente. La respuesta del biosensor fue máxima a $600 \mathrm{mV}$, su tiempo de respuesta fue de 20 segundos en un intervalo de concentración de respuesta lineal de 0.1 a $5 \mathrm{mM}$ de glucosa y sensibilidad de $1.4 \mu \mathrm{A} / \mathrm{mM}$. La estabilidad y vida útil del biosensor desarrollado dependen de la frecuencia de uso y el biocompósito actúa como reservorio de enzimas y de mediador electroquímico.
\end{abstract}

Palabras clave: método analítico, biosensor, glucosa, platino, voltamperometría

\section{Glucose Biosensor based on a Graphite-Epoxy- Platimum- Glucose Oxidase dispersed Biocomposite}

\begin{abstract}
The aim of this work was to develop a glucose biosensor based on electrochemical properties of a graphite-epoxy-platinum- glucose oxidase composite. The food and beverage industry demand fast, precise and reliable analytical methods to evaluate and to assure the quality of its products and to optimize its processes. The development of amperometric enzyme biosensors represents a viable option that satisfies these requirements. The working potential and the characterization of the response of the biosensor were determined by cyclic voltammetry and amperometry, respectively. The biosensor response was maximum at $600 \mathrm{mV}$, with a response time of 20 seconds within the concentration range of linear response from 0.1 to $5 \mathrm{mM}$ glucose and sensitivity of $1.4 \mu \mathrm{A} / \mathrm{mM}$. The stability and lifetime of the proposed biosensor depend on the frequency of use, where the biocomposite acts as a reservoir of enzymes and electrochemical mediator.
\end{abstract}

Keywords: analytical method, biosensor, glucose, platinum, voltammetry

Información Tecnológica Vol. 22 No 1 - 2011 


\section{INTRODUCCION}

La necesidad de contar con resultados analíticos precisos y confiables para la monitorización y el control de procesos químicos, ha originado el desarrollo de nuevos y sofisticados métodos de análisis que requieren de una costosa instrumentación, instalación y mantenimiento, lo que ha dado lugar a la aparición de una sub-disciplina de la química analítica que es, la química analítica de procesos, cuyo objetivo principal consiste en suministrar información cualitativa y cuantitativa de un determinado proceso químico en función del tiempo; información que además de permitir la monitorización y el control de los parámetros de calidad, también es útil para optimizar de manera eficiente y desde el punto de vista industrial, el consumo de energía y de materias primas, así como la duración del proceso y el aspecto final del producto terminado (Riebe, 1990; Ferreira et al., 2003).

Aunado a ello, se han desarrollado también pequeños, sencillos y versátiles dispositivos que permiten simplificar la adquisición de información analítica, sin renunciar a la selectividad y sensibilidad que brindan los poderosos métodos instrumentales de análisis. Se trata de los sensores químicos, simples dispositivos analíticos que responden selectiva y cuantitativamente a un tipo de analito en particular, a través de una reacción química en la que se generan señales primarias de reconocimiento, las cuales posteriormente son convertidas en señales eléctricas a través de un transductor (Gómez y Alegret, 1997; Kissinger, 2005). Los sensores químicos están constituidos por dos partes principales: un elemento de reconocimiento molecular o receptor que sólo reconoce al analito por cuantificar, y un elemento transductor de la señal de reconocimiento, en cuya superficie se encuentra inmovilizado el receptor (Fig. 1) (Thevenot et al., 2001).

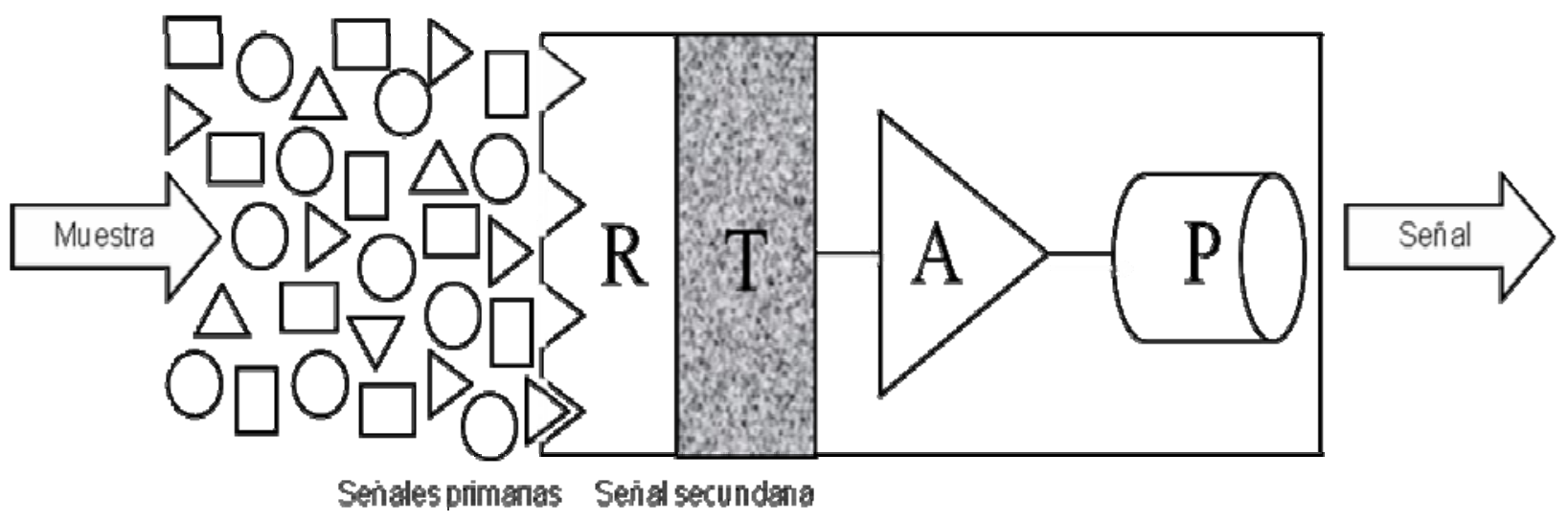

Fig. 1: Diagrama esquemático del funcionamiento de un sensor químico

El sistema de reconocimiento o receptor $(R)$ sólo reconoce el analito por cuantificar $(\Delta)$ presente en la muestra. La señal generada en el proceso de reconocimiento es convertida en una señal eléctrica mediante el transductor $(T)$, la cual posteriormente es amplificada ( $A$ ) y finalmente procesada y presentada en forma digital $(P)$. El receptor puede interactuar con el analito a través de mecanismos físicos, químicos o biológicos.

La escasa versatilidad en la disponibilidad de receptores sintéticos es determinante para que los sensores químicos presenten un grado de reconocimiento limitado, lo que originó el uso de materiales biológicos como elementos de reconocimiento molecular, mucho más selectivos y versátiles que los de tipo sintético. A estos sensores químicos que incorporan materiales biológicos como elementos de reconocimiento molecular, se les dio el nombre de biosensores (Wang, 2001; Sharma et al., 2003; Kissinger, 2005). Debido a su alta selectividad y sensibilidad, las enzimas destacan como los elementos de reconocimiento molecular más utilizados en la construcción de biosensores (Dornelles y Tatsuo, 2002, Leonard et al., 2003).

El primer biosensor fue un analizador de glucosa desarrollado por Clark y Lyons en 1962 y comercializado a partir de 1975 por Yellow Springs Instrument Company. Este biosensor se denominó "enzyme electrode" y consistía en un dispositivo que contenía la enzima glucosa 
oxidasa acoplada a un electrodo para oxígeno (Chaubey y Malhotra, 2002). Desde entonces, el desarrollo de los biosensores se ha centralizado en el campo del diagnostico clínico debido a lo cual, el biosensor de glucosa ha sido el biosensor más desarrollado a nivel mundial (Wang, 2001, Newman y Turner, 2005; Kissinger, 2005). Gracias al apoyo de los instrumentos de la biotecnología y a la investigación postgenómica, los biosensores actualmente suponen potentes herramientas de análisis que encuentran numerosas aplicaciones en la industria agroalimentaria medioambiental, químico, farmacéutico y militar (Rekha et al., 2000; Patel, 2002; Ferreira et al., 2003). En la industria agroalimentaria, las tres grandes áreas de aplicación de los biosensores son: seguridad alimentaria, calidad alimentaria y control de procesos (González et al., 2005).

Las características más destacables que convierten a los biosensores en opciones altamente atractivas para competir en el mercado con otras tecnologías son: su especificidad, su alta sensibilidad, su corto tiempo de análisis, su capacidad de inclusión en sistemas integrados, su facilidad de automatización, su capacidad de trabajar en tiempo real, su versatilidad que permite el diseño de dispositivos a la carta, y su bajo coste, entre otras (González et al., 2005)

Desde sus orígenes y hasta la actualidad se han desarrollado diferentes configuraciones del biosensor de glucosa, en la mayoría de ellas se utiliza la enzima glucosa oxidasa (GOD) inmovilizada de diversas formas y empleando diferentes sistemas de transducción de la señal (Wang, 2001; Patel, 2002; Nakamura y Karube, 2003; Zhou et al., 2008). La reacción básica catalizada por esta enzima es la siguiente:

$$
\text { Glucosa }+\mathrm{O}_{2} \longrightarrow \text { AOD } \longrightarrow \text { Acido glucónico }+\mathrm{H}_{2} \mathrm{O}_{2}
$$

Estos prototipos presentan un reducido intervalo de concentración de respuesta lineal, así como un corto tiempo de vida media debido a la constante pérdida de material sensor (enzima y mediador electroquímico) desde el cuerpo del biosensor al seno de la solución (Chaubey y Malhotra, 2002; Dornelles y Tatsuo, 2002; Shan et al., 2007). Es por ello que, el objetivo del presente trabajo consistió en desarrollar un biosensor de glucosa de matriz de fase sólida, en donde se aprovechan las propiedades electroquímicas del platino para regenerar la enzima glucosa oxidasa y para conducir el flujo de electrones desde la enzima hacia la superficie del electrodo del amperímetro, buscando con ello mejorar el intervalo de respuesta y la estabilidad del biosensor.

\section{MATERIALES Y MÉTODOS}

\section{Materiales}

Resina EpoTek-H77 (Epoxy Technology, USA), grafito en polvo (BDH Laboratory Supplies, England), platino en polvo (Sigma-Aldrich, USA) y glucosa oxidasa (EC 1.1.3.4) con $160 \mathrm{UA} / \mathrm{mg}$ de sólido (Sigma, USA), Frudex 55, 70, 80 y 90 (Arancia, México). Glucosa, fructosa, sacarosa, maltosa, lactosa, galactosa, ácido ascórbico, ácido cítrico y ácido málico (Sigma, USA). El, resto de los reactivos fueron grado analítico y en la preparación de las soluciones se utilizó agua bidestilada.

\section{Construcción del Biosensor}

La construcción del biosensor de glucosa se basó en el modelo descrito por Gómez y Alegret, (1997). El biosensor está formado por dos partes: el biocompósito y el cuerpo. El biocompósito está constituido por una fase conductora de la electricidad (grafito), dispersa en una fase polimérica (resina epoxi) que aglutina e inmoviliza los constituyentes del sistema sensor integrados por la enzima glucosa oxidasa y el platino en polvo como mediadores redox. La proporción en que se combinan estos constituyentes es 63:17:17:3. Por su parte, el cuerpo del biosensor consta de un tubo de PVC en el que se introduce un conector eléctrico unido a una lámina de cobre (Fig. 2). El hueco entre el tubo y la lámina se rellena con el biocompósito y se somete a un curado térmico a $40^{\circ} \mathrm{C} / 6$ días, al cabo de los cuales la superficie del biosensor es pulida con papel alúmina (Orion, USA). El biosensor se mantuvo en refrigeración a $4^{\circ} \mathrm{C}$ cuando no era utilizado. 


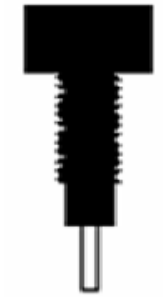

Conectrr herrora

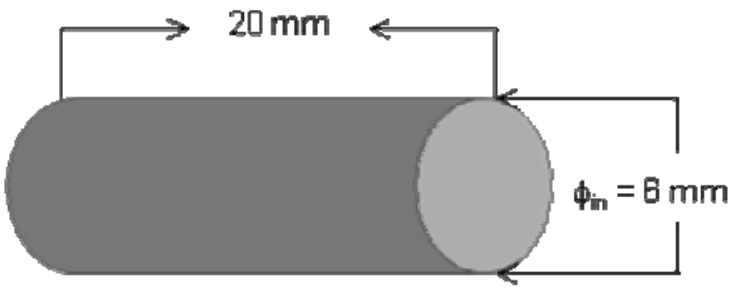

Cuerpo del biosensor tubo de PVC

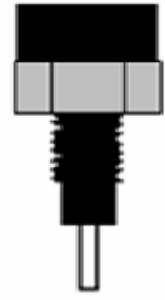

Conector y tuerca

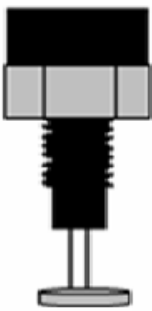

Conectar tuercay lámina de Cu

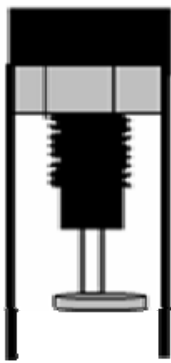

Conector dentra del tuba de PVC

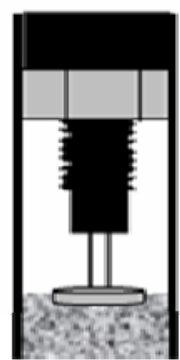

Conector

mas

biocompásitn

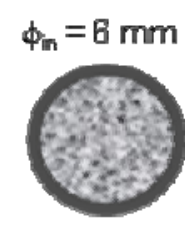

Superficie del biosensar

Fig. 2: Construcción del cuerpo del biosensor

\section{Caracterización del Biosensor}

El potencial de trabajo del biosensor se determinó por voltamperometría de barrido lineal en un analizador electroquímico Autolab PGSTAT20 (Eco Chemie, Holanda). El barrido de potencial se realizó entre 0 a $800 \mathrm{mV}$ y a una velocidad de $50 \mathrm{mV} / \mathrm{s}$. Inicialmente el barrido se realizó sobre el electrolito soporte (solución reguladora de fosfatos $0.1 \mathrm{M}$ más $\mathrm{KCl} 0.1 \mathrm{M}$ a pH de 7.5 y $25^{\circ} \mathrm{C}$ ) y posteriormente sobre soluciones de glucosa de diferente concentración; todas ellas preparadas con el mismo electrolito soporte como disolvente. Una vez conocido el potencial de trabajo se realizó la caracterización de la respuesta del biosensor a diferentes condiciones de trabajo, para ello se utilizó un detector amperométrico LC-4C (Bioanalytical Sistems Inc. USA). En todos los casos, las determinaciones se realizaron en una celda electroquímica de $50 \mathrm{~mL}$ provista de doble pared para control de temperatura y a un $\mathrm{pH}$ de 7.5 y temperatura de $25^{\circ} \mathrm{C}$, excepto cuando se estudia el efecto del $\mathrm{pH}$ y la temperatura sobre la respuesta del biosensor, respectivamente. De esta forma se determinó el efecto del tiempo de reacción, el efecto del $\mathrm{pH}$, el efecto de la temperatura y de la concentración de sustrato sobre la respuesta del biosensor desarrollado (Habermüller et al., 2000; Rekha et al., 2000). Finalmente también se evaluó la estabilidad de la respuesta del biosensor con respecto al tiempo; para ello se hicieron evaluaciones diarias durante los primeros quince días después de su construcción, posteriormente las determinaciones se hicieron cada cinco días durante un mes y por ultimo cada quince días durante cuatro meses. El biosensor se utilizó como electrodo de trabajo frente a un electrodo de referencia de $\mathrm{Ag} / \mathrm{AgCl}$ (Orion USA), y un electrodo auxiliar de platino modelo 108053117 (Ingold, Suiza). La superficie del biosensor fue pulida entre determinaciones sucesivas y las lecturas fueron tomadas un minuto después de adicionar el sustrato. Todas las determinaciones se realizaron por triplicado y puliendo la superficie del electrodo entre determinaciones sucesivas.

\section{Análisis estadístico de los resultados}

Los datos reportados corresponden a la media aritmética de tres determinaciones sucesivas puliendo la superfície del biosensor entre una determinacion y otra. Adicionalmente se realizaron análisis de varianza (ANOVA) y se identificaron las diferencias en los valores promedio mediante la prueba de Duncan con un factor de confianza del 95\%, utilizando el software estadístico SPSS versión 9.0 para Windows (Fry, 1996). 


\section{RESULTADOS Y DISCUSIÓN}

\section{Determinación del potencial de trabajo $(E)$}

El biosensor desarrollado respondió a la concentración de glucosa en el medio de reacción y su respuesta fue máxima a un potencial de trabajo de $600 \mathrm{mV}$ (Fig. 3). Este potencial de trabajo del biosensor de glucosa desarrollado es relativamente elevado comparado con el de biosensores de segunda o tercera generación (Habermüller et al., 2000, Newman y Turner, 2005), lo que conlleva a posibles interferencias de especies electroactivas como el ácido ascórbico, las cuales pueden estar presentes en muestras reales (Dornelles y Tatsuo, 2002).
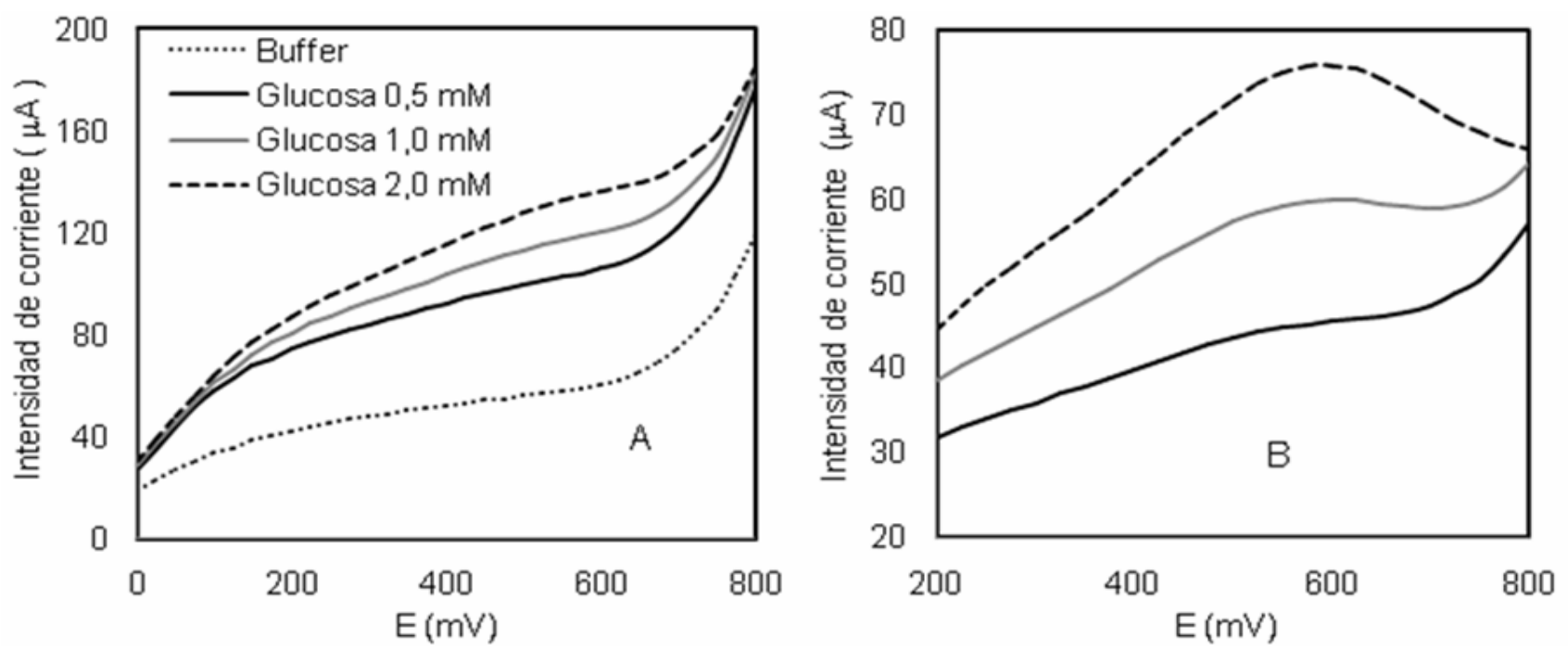

Fig. 3: A: Voltamperometría de barrido lineal del biosensor. B: Señal de las soluciones de glucosa menos señal de solución tampón entre $200-800 \mathrm{mV}$. Condiciones: $\mathrm{pH} 7.5$ y $25^{\circ} \mathrm{C}$.

\section{Estimación del tiempo de respuesta}

A partir del momento en que se adiciona la glucosa a la celda electroquímica, el tiempo mínimo requerido para alcanzar la estabilidad de la señal del biosensor fue de 20 segundos; es decir, para llegar al 95\% de la intensidad máxima de la señal. Este tiempo de respuesta es similar al que presentan algunos biosensores reportados en la literatura (Zhang et al., 2010). No hubo diferencias estadísticas significativas en la intensidad de corriente registrada a mayores tiempos de respuesta y a las diferentes concentraciones de glucosa ensayadas (Fig. 4).

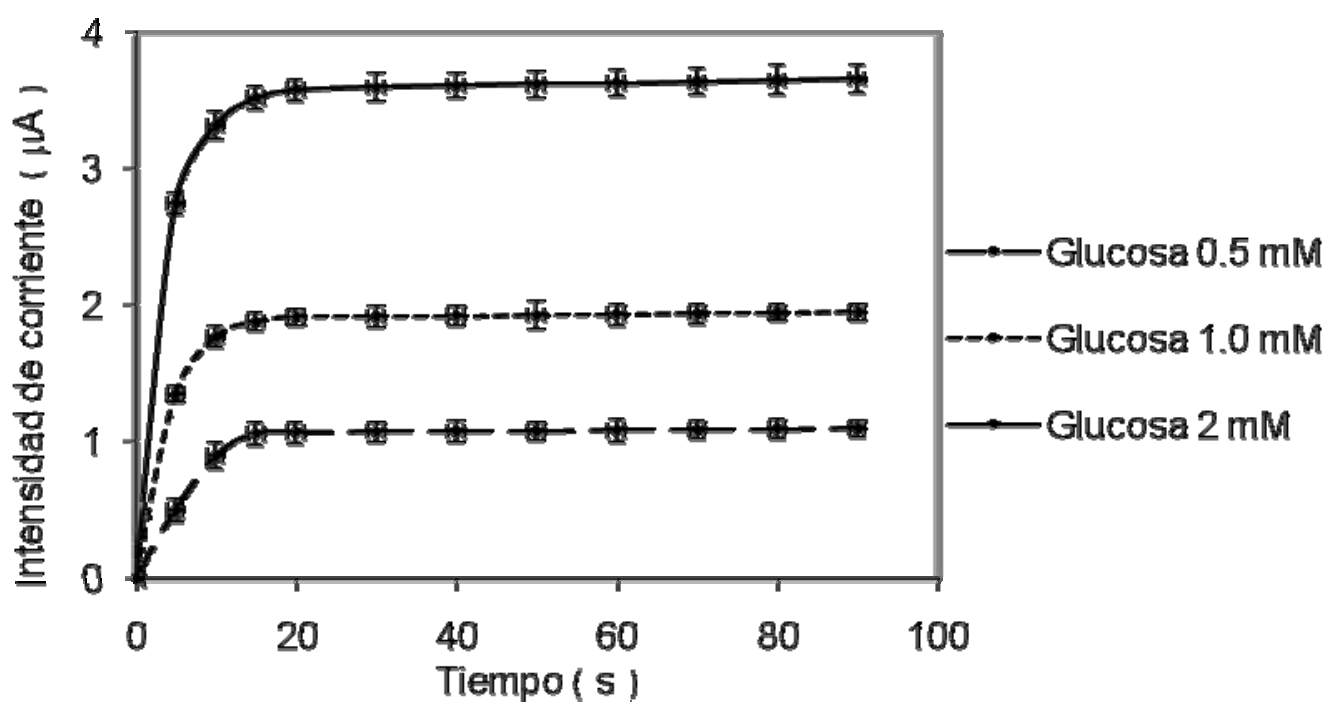

Fig. 4: Variación de la respuesta del biosensor de glucosa con respecto al tiempo y a diferentes concentraciones de glucosa. Condiciones: $\mathrm{E}=600 \mathrm{mV}, \mathrm{pH}=7.5$ y $\mathrm{T}=25^{\circ} \mathrm{C}$. 


\section{Efecto de la Temperatura y el $\mathrm{pH}$}

Dado que la actividad de una enzima y por lo tanto la respuesta y la estabilidad de un biosensor enzimático dependen del $\mathrm{pH}$ y de la temperatura del medio de reacción, la temperatura y el $\mathrm{pH}$ de trabajo de un biosensor debe cumplir con dos requisitos: proporcionar la máxima sensibilidad y estabilidad al biosensor (Retama et al., 2005; Ricci et al., 2007). La respuesta del biosensor de glucosa fue máxima a una temperatura de $30^{\circ} \mathrm{C}$ (Fig. 5-A) y a pH de 7.5 (Fig. 5-B). A mayores valores de temperatura y $\mathrm{pH}$ se inicia la inactivación de la enzima y por ende, disminuye la respuesta del biosensor. La intensidad de la respuesta del biosensor se incrementó en 1.87 veces dentro del intervalo de temperatura comprendido entre $20-30^{\circ} \mathrm{C}$. Por lo anterior, con objeto de garantizar la estabilidad del biosensor desarrollado, todas las determinaciones posteriores se realizaron a un $\mathrm{pH}$ de 7.5 y a una temperatura de $25^{\circ} \mathrm{C}$.

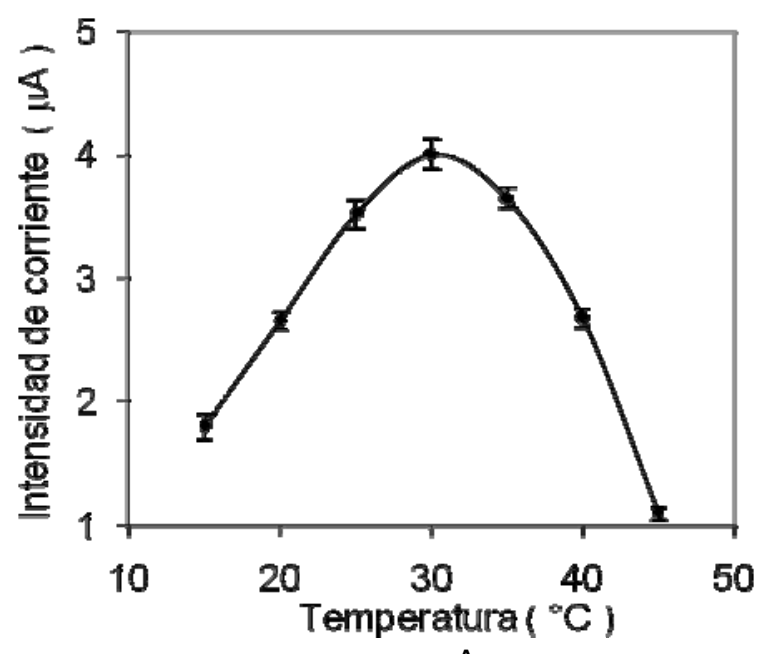

A

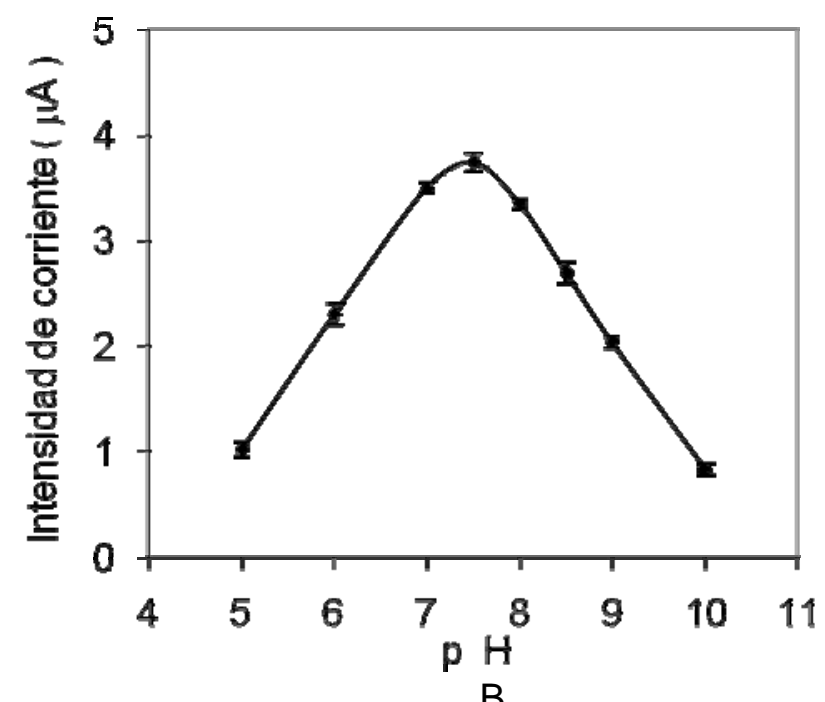

Fig. 5: Efecto de la temperatura (A) y efecto del $\mathrm{pH}(\mathrm{B})$ en la respuesta del biosensor de glucosa. Condiciones: $\mathrm{E}=600 \mathrm{mV}$, concentración de glucosa $2 \mathrm{mM}, \mathrm{pH}=7.5$ y $\mathrm{T}=25^{\circ} \mathrm{C}$.

Efecto de la concentración de glucosa

La respuesta del biosensor fue directamente proporcional a la concentración de glucosa dentro del un intervalo de concentraciones comprendido entre 0.1 a $5 \mathrm{mM}$ de glucosa $(r=0.9925$ para $n=3$ determinaciones)(Fig. 6).

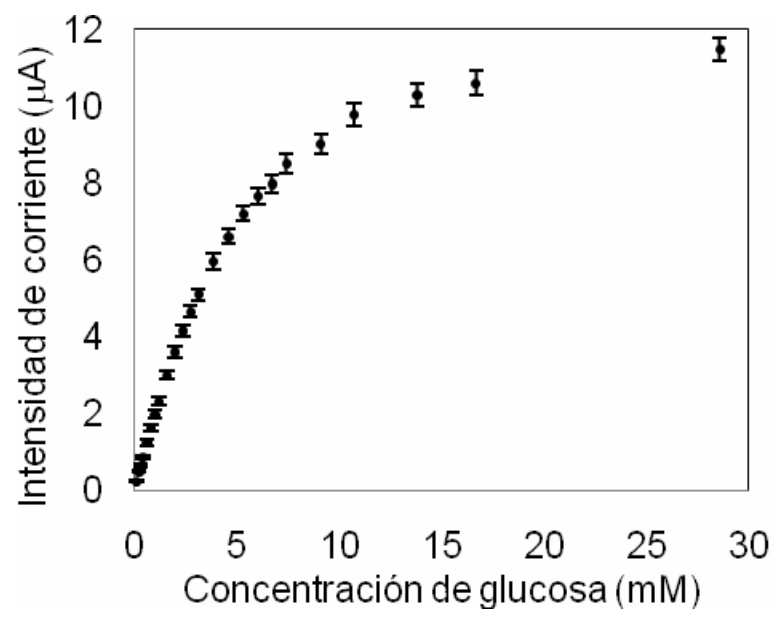

A

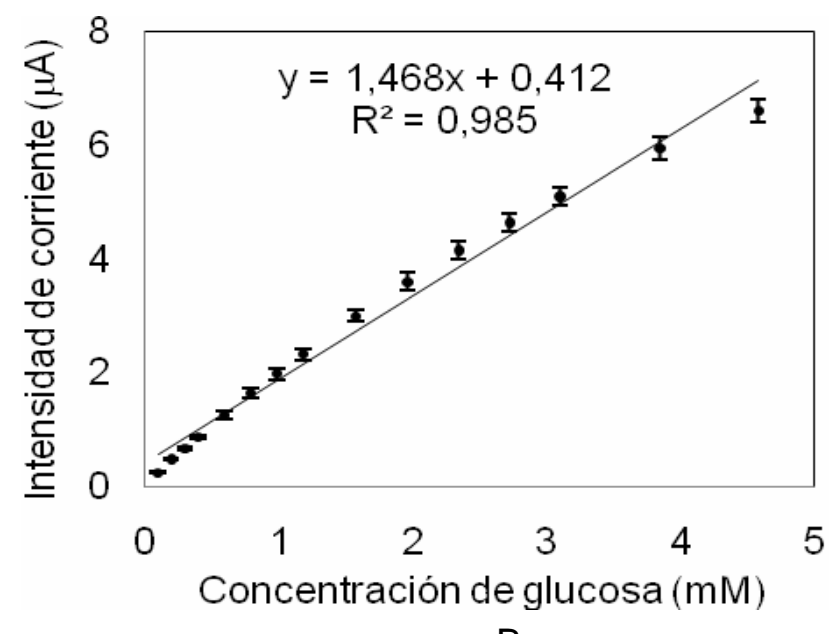

$B$

Fig. 6: Respuesta del biosensor en función de la concentración de glucosa $(A)$ e intervalo de concentración de respuesta lineal del biosensor (B): Condiciones: $E=600 \mathrm{mV}, \mathrm{pH}=7.5$ y $\mathrm{T}=25^{\circ} \mathrm{C}$. 
Dentro del intervalo de concentraciones de respuesta lineal (de 0.1 a $5 \mathrm{mM}$ ), la máxima sensibilidad del biosensor fue de $1.468 \mu \mathrm{A} / \mathrm{mM}$ y su límite de detección inferior fue de $0.05 \mathrm{mM}$, el cual se obtuvo a una relación señal:ruido de 3.0, mientras que el límite de detección superior se establece a través de la curva de calibración y en este caso fue de $5.0 \mathrm{mM}$ de glucosa y corresponde a la concentración de glucosa donde el valor del coeficiente de correlación de la curva de calibración es mayor de 0.99; en nuestro caso, $r=0.9925$. Al comparar este intervalo de operación del biosensor desarrollado con los que se encuentran reportados en la literatura para biosensores similares, podemos darnos cuenta de que dichos intervalos de operación dependen tanto del sistema de transducción de la señal empleado como del diseño mismo del biosensor; es decir, de su configuración (Dorneles y Tatsuo 2002; Ricci et al., 2005; Njagi y Andreescu, 2007; Pemberton et al., 2009; Zhang et al., 2010). Desde el punto de vista operativo, es importante que los biosensores presenten un amplio intervalo de concentraciones de respuesta lineal, especialmente cuando se pretende que operen en procesos continuos, donde no es posible ajustar las concentraciones de los analitos dentro de los intervalos de respuesta lineal de dichos instrumentos (Rekha et al., 2000), pero en el caso de operar en procesos discontinuos o por lote, resulta irrelevante que tan amplio sea este intervalo de concentraciones de respuesta lineal del biosensor, puesto que aun así es necesario ajustar la concentración del analito en las muestras por analizar, a fin de que dicha concentración caiga dentro del intervalo de respuesta lineal del biosensor (Ferreira et al., 2003; Montañez et al., 2006).

\section{Reproducibilidad de la señal}

La reproducibilidad de la respuesta del biosensor se determinó en dos modalidades; una de ellas fue puliendo su superficie con papel alúmina entre una determinación y otra, mientras que la otra se hizo sin pulir su superficie entre determinaciones sucesivas. La utilización continua del biosensor se ve acompañada de una notable disminución de su sensibilidad, cuando su superficie no es pulida entre una calibración y otra, a tal grado que después de 10 calibraciones sucesivas (Fig. 7, calibraciones 1-10), el biosensor de glucosa mantiene sólo el 25\% de su sensibilidad inicial; pero dicha sensibilidad se recupera al $100 \%$ y se mantiene prácticamente constante ( $D E R=1.14$ para $n=10$ ) mediante un simple pulido de su superficie con papel de alúmina (Fig. 7: calibraciones 11-20); lo cual es indicativo de la homogeneidad en la mezcla del biocompósito, puesto que en cada pulida del biosensor se renueva la superficie sensitiva integrada por la enzima y el elemento transductor de la señal.

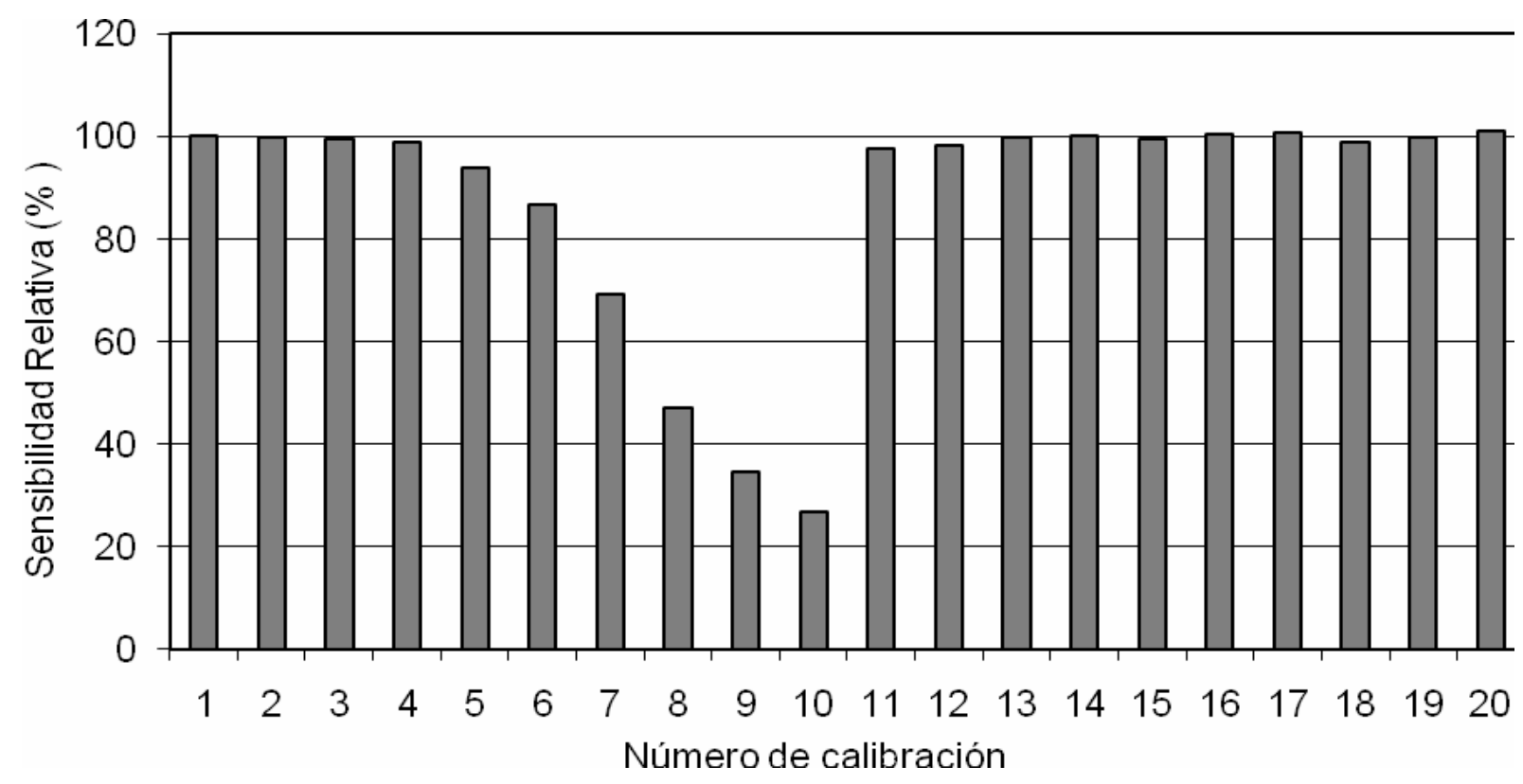

Fig. 7: Reproducibilidad de la señal del biosensor sin pulir (calibraciones 1-10), y puliendo (calibraciones 11-20) la superficie del biosensor entre determinaciones sucesivas). Condiciones: $\mathrm{E}=600 \mathrm{mV}, \mathrm{pH}=7.5, \mathrm{~T}=25^{\circ} \mathrm{C}$ y concentración de glucosa $2 \mathrm{mM}$. 
La disminución de la sensibilidad del biosensor de glucosa cuando no se pule su superficie entre una calibración y otra, puede atribuirse a la pérdida de la enzima que se encuentra en la superficie del biosensor debido a fenómenos de solubilidad o bien, por una desnaturalización progresiva de la misma; fenómeno que ya ha sido observado por otros investigadores (Montañez et al., 2006).

\section{Estabilidad y vida de operación}

La sensibilidad del biosensor de glucosa se mantuvo prácticamente constante durante los primeros 120 días (Fig. 8), ya que durante este periodo no se presentó una diferencia estadística significativa en la sensibilidad relativa del biosensor, lo cual es apoyado por el relativamente bajo valor de la desviación estándar relativa de la serie (DER=1.26\% para $n=51)$.

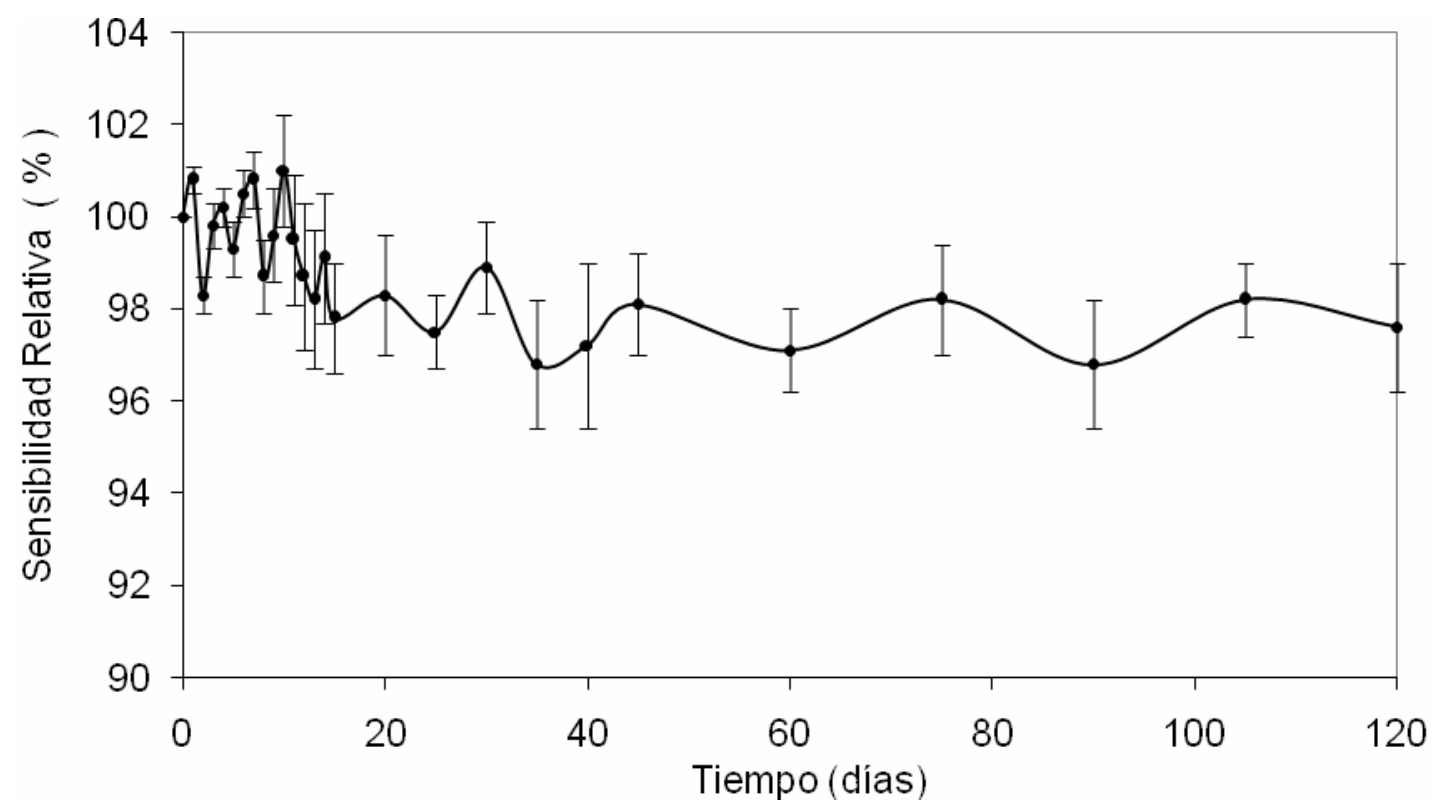

Fig 8: Variación de la sensibilidad del biosensor de glucosa con respecto al tiempo. Condiciones: $\mathrm{E}=600 \mathrm{mV}, \mathrm{pH}=7.5, \mathrm{~T}=25^{\circ} \mathrm{C}$ y concentración de glucosa $2 \mathrm{mM}$.

Después 24 meses el biosensor de glucosa desarrollado aún conserva el 85\% de su sensibilidad inicial; es decir, durante ese tiempo su sensibilidad ha disminuido sólo en un 15\%. Esta pérdida de sensibilidad del biosensor de glucosa con respecto al tiempo, podría deberse a una inactivación progresiva de la enzima que se encuentra inmovilizada en el biocompósito; de aquí la importancia de almacenar el biosensor en refrigeración a $4^{\circ} \mathrm{C}$ cuando no se está utilizando.

De acuerdo con estos resultados, podemos decir que el biocompósito actúa como reservorio de enzima y de mediador electroquímico, por lo que la vida útil de éstos dispositivos queda determinada por el tiempo que tarde en agotarse el material sensor que integra el biocompósito, y este a su vez, de la frecuencia con que se utilice el biosensor, ya que entre una determinación y otra, cada vez que se pule la superficie del biosensor se va perdiendo parte del material sensor que integra el biocompósito (Montañez et al., 2006).

\section{Selectividad de la respuesta del biosensor}

La selectividad se refiere a la capacidad del biosensor para evitar la respuesta que pueden causar sustancias interferentes, ya sea que interfieran con la enzima del biosensor, o bien, sustancias electroactivas que puedan sufrir reacciones de oxido-reducción al potencial de trabajo del biosensor (Rekha, 2000). Para determinar la selectividad del dispositivo, la respuesta del biosensor de glucosa desarrollado se midió en presencia de algunas posibles sustancias interferentes tales como fructosa, sacarosa, maltosa, galactosa, lactosa, ácido ascórbico, ácido 
cítrico y ácido málico. Las determinaciones se realizaron a un potencial de trabajo de $\mathrm{E}=600 \mathrm{mV}$ y empleando un electrodo de $\mathrm{Ag} / \mathrm{AgCl}$ como electrodo de referencia; como electrolito soporte se utilizó una solución reguladora de fosfatos $0.1 \mathrm{M}$ más $\mathrm{KCl} 0.1 \mathrm{M}$ a pH de 7.5 y $25^{\circ} \mathrm{C}$ y a una concentración de glucosa $2 \mathrm{mM}$ e igual concentración de las sustancias interferentes (Montañez et al., 2006). En presencia de cualquiera de las posibles sustancias interferentes, el biosensor respondió selectivamente a la glucosa, que es el sustrato natural de la enzima glucosa oxidasa, pero su respuesta se vio notablemente alterada en presencia del ácido ascórbico, el cual causó un incremento del $8.5 \pm 0.6 \%$ en la respuesta del biosensor de glucosa. Esto demuestra que por el lado de la acción de la enzima el biosensor es altamente selectivo, propiedad que le confiere la enzima glucosa oxidasa utilizada como elemento de reconocimiento molecular. El inconveniente surge por el lado del potencial de trabajo del biosensor, el cual es mayos que el potencial redox de algunas especies electroactivas como el ácido ascórbico, el cual puede encontrarse en muestras reales.

\section{Determinación del contenido de glucosa en muestras reales}

El contenido de glucosa en cuatro diferentes jarabes de maíz de alto contenido de fructosa (Frudex 55, 70, 80 y 90) fue determinado con el biosensor de glucosa desarrollado y por medio de la cromatografía de líquidos de alta presión (Versele y Van Dame, 1987). Para ello se utilizó un Cromatografo de Líquidos LDC modelo 3200 (Variant, USA) equipado con un detector de índice de refracción modelo 350 (Variant, USA) y una bomba LDC Analytical. Se utilizó una columna SPHEREX $5 \mathrm{NH} 2$ de $250 \mathrm{~mm}$ de longitud y $4.6 \mathrm{~mm}$ de diámetro. Como fase móvil se utilizó una solución de acetonitrilo:agua en relación 85:15. Las condiciones de trabajo fueron: velocidad de flujo de $0.4 \mathrm{~mL} / \mathrm{min}$, temperatura de $35^{\circ} \mathrm{C}$ y los estándares se inyectaron a una concentración de 2 $\mathrm{mg} / \mathrm{mL}$. Los resultados promedio de tres determinaciones sucesivas obtenidos por ambos métodos se muestran en la tabla 1.

Tabla 1: Contenido de glucosa determinado por HPLC y con el biosensor de glucosa desarrollado

\begin{tabular}{cccc}
\hline Muestra & $\begin{array}{c}\text { HPLC } \\
\text { Glucosa }(\mathrm{g} / 100 \mathrm{~g})\end{array}$ & $\begin{array}{c}\text { Biosensor } \\
\text { Glucosa }(\mathrm{g} / 100 \mathrm{~g})\end{array}$ & $\begin{array}{c}\text { Desviación estándar } \\
\text { relativa (\%) }\end{array}$ \\
\hline Frudex 55 & $46.84 \pm 0.75$ & $46.76 \pm 0.25$ & 1.55 \\
Frudex 70 & $30.75 \pm 0.56$ & $30.45 \pm 0.36$ & 1.25 \\
Frudex 80 & $20.65 \pm 0.35$ & $20.50 \pm 0.28$ & 1.15 \\
Frudex 90 & $10.35 \pm 0.45$ & $10.15 \pm 0.55$ & 1.05 \\
\hline
\end{tabular}

No existe diferencia estadística significativa en los resultados de concentración de glucosa obtenidos por ambos métodos analíticos para cada uno de los cuatro diferentes jarabes de maíz con alto contenido de fructosa analizados y en todos los casos, se obtuvo una desviación estándar relativa inferior al $2 \%$, lo cual es indicativo de la alta correlación que existe entre los resultados obtenidos de concentración de glucosa obtenidos con el biosensor desarrollado, y los proporcionados por la cromatografía de líquidos de alta presión como método de referencia.

\section{CONCLUSIONES}

El alto potencial de trabajo $(E=600 \mathrm{mV}$ ) requerido por el biosensor desarrollado implica el tener que trabajar con muestras reales libres de especies electroactivas que puedan interferir en la respuesta del dispositivo.

El biocompósito constituye una mezcla homogénea de material sensor, pues no existen diferencias estadísticas significativas entre las respuestas del biosensor cuando su superficie es pulida entre una determinación y otra.

Su reducido tiempo de respuesta y su amplio intervalo de concentraciones de respuesta lineal, convierten a éste biosensor en una prometedora herramienta analítica para el monitoreo y control de procesos donde existe la necesidad de cuantificar la glucosa. 
La aplicación del biosensor desarrollado proporciona datos analíticos similares a los obtenidos por cromatografía de líquidos de alta presión al cuantificar el contenido de glucosa en muestras reales.

El dispositivo responde selectivamente a la concentración de glucosa presente en la muestra, excepto cuando en la misma se encuentra el ácido ascórbico

La vida útil del biosensor está determinada por su frecuencia de uso, ya que el biocompósito actúa como reservorio de enzima y mediador electroquímico para sucesivas aplicaciones.

\section{REFERENCIAS}

Chaubey, A. y B. Malhotra, Review of Mediated Biosensors. Biosensors \& Bioelectronics: 17, 441456 (2002).

Dornelles, M.L. y K.L. Tatsuo, Review of the use of biosensors as analytical tools in the food and drink industries. Food Chemistry, 77, 237-256 (2002).

Ferreira, S., M.B. De Souza, J.O. Trierweiler, R.O.M. Folly y B. Hitzmann, Aspects concerning the use of biosensors for process control: experimental and simulation investigations. Computers \& Chemical Engineering, 27, 1165-1173 (2003).

Fry, J., Biological data analysis. A practical approach, 418-420. Oirc Press. USA (1996).

Gomez, M.R. y S.S. Alegret, Los sensores químicos: una aportación a la instrumentación analítica. Educación Química, 8 (4), 191-196 (1997).

González, R. V., I.E. García, G.O. Ruiz y C.L. Gago, Aplicaciones de los biosensores en la industria alimentaria. 5-24. Elecé Industria Gráfica. Madrid, España. (2005).

Habermüller, K., M. Mosbach y W. Schuhmann, Review: Electron-transfer mechanisms in amperometric biosensors. J. Anal. Chem., 366, 560-568 (2000).

Kissinger, P.T., Biosensors: a perspective. Biosensors and Bioelectronics 20, 2512-2516 (2005).

Leonard, P., S. Hearty, J. Brennan, L. Dunne, J. Quinn, T. Chakraborty y R. Kennedy, Review: Advances in biosensors for detection of pathogens in food and water. Enzyme and Microbial Technology, 32, 3-13 (2003).

Montañez, S.J.L., S.S. Alegret, M.J.A. Salazar y R.E.G. Ramos, A new amperometric biosensor for fructose determination based on epoxy-graphite-TTF-TCNQ-FDH biocomposite. Eur Food Res Technol, 223, 379-386 (2006).

Nakamura, $\mathrm{H}$ y I. Karube, Current research activity in biosensors. Analytical \& Bioanalytical Chemistry, 377, 446-468 (2003).

Newman, J.D., y A.P.F. Turner, Home blood glucose biosensors: a commercial perspective. Biosensors and Bioelectronics 20: 2435-2453 (2005).

Njagi, J., y S. Andreescu, Stable enzyme biosensors based on chemically synthesized Aupolypyrrole nanocomposites. Biosensors and Bioelectronics 23, 168-175 (2007).

Patel, P.D., Biosensors for measurement of analytes implicated in food safety: a review. Trends in Analytical Chemistry, 21 (2), 96-115 (2002). 
Pemberton, R.M., R. Pittson, N. Biddle y J.P.Hart. Fabrication of microband glucose biosensors using a screen-printing water-based carbon ink and their application in serum analysis. Biosensors and Bioelectronics 24, 1246-1252 (2009).

Rekha, K., S. Thakur y N.G. Karanth, Biosensors for the detection of organophosphorous pesticides. Critical Reviews in Biotechnology, 20 (3), 213-235 (2000).

Retama, J. R., M.S. Paniagua, J.P. Hervás, G.F. Cabanillas, E.L. Cabarcos y B.L. Ruiz, Biosensors based on acrylic microgels. A comparative study of immobilized glucose oxidase and tyrosinase. Biosensors and Bioelectronics 20, 2268-2275 (2005).

Riebe, M.T. y J.D. Eustace, Process analytical chemistry: an industrial perspective. Anal. Chem., 62(2), 65-71 (1990).

Ricci F., D. Mosconea, C. Tutaa, G. Palleschia, A. Aminec y A. Posciab, Novel planar glucose biosensors for continuous monitoring use. Biosensors and Bioelectronics 20, 1993-2000 (2005).

Ricci , F., F. Caprio, A. Poscia, F. Valgimigli, D. Messeri, E. Lepori y G. Dall'Oglio, Toward continuous glucose monitoring with planar modified biosensors and microdialysis Study of temperature, oxygen dependence and in vivo experiment. Biosensors and Bioelectronics 22, 20322039 (2007).

Shan, D., M. Zhua, H. Xuea y Cosnier, S., Development of amperometric biosensor for glucose based on a novel attractive enzyme immobilization matrix: Calcium carbonate nanoparticles. Biosensors and Bioelectronics, 22, 1612-1617 (2007).

Sharma, S.K., N. Sehgal y A. Kumar, Biomolecules for development of biosensors and their applications. Current Applied Physics, 3, 307-316 (2003).

Thevenot, R.D., K. Toth, R. Durst y G. Wilson, Electrochemical biosensors: recommended definitions and classification. Biosensors \& Bioelectronics, 16, 121-131 (2001).

Verzele, M. y F. Van Damme, A critical review of some liquid chromatography systems for the separation of sugars. Journal of Chromatography, 362, 23-27 (1987).

Wang, J., Glucose biosensors: 40 years of advances and challenges. Electroanalysis, 13(12), 983988 (2001).

Zhang, Z.B., S.J. Yuan, X.L. Zhu, K.G. Neoh, E.T. Kang, Enzyme-mediated amperometric biosensors prepared via successive surface-initiated atom-transfer radical polymerization. Biosensors and Bioelectronics 25, 1102-1108 (2010).

Zhou M., L. Shang, B. Li, L. Huang y S. Dong, Highly ordered mesoporous carbons as electrode material for the construction of electrochemical dehydrogenase- and oxidase-based biosensors. Biosensors and Bioelectronics 24, 442-447 (2008). 
